\title{
Guest Editorial: Smart Transportation Based on Multimedia Data Mining
}

\author{
Zhihan Lv ${ }^{1}$ - Chen Zhong $^{2} \cdot$ Dingde Jiang $^{3}$
}

Published online: 6 October 2016

(C) Springer Science+Business Media New York 2016

The advent of new technologies such as the Internet of Things (IoT) and cloud computing has brought opportunities for the development of Smart Transportation. In line with the essential of "smart cities", smart transportation highlights the use of data. In particular, data are captured in large quantity, from multi-source, and in real-time. Moreover, Smart Transportation lays greater emphasis on knowledge discovery, information sharing and supported decision making. This new notion involves some tasks in which traditions are replaced by intelligent technology requiring manual discrimination and resolution to reach the optimization. In addition, with the development of Internet of Vehicles, Smart Transportation pays greater attention to the interconnection between transportation system and other information systems to the maximum extent possible. Then it can be seen that further efficient management and deeper analysis of transportation data are the key tasks in developing smart transportation.

We have finally selected 15 manuscripts for this special issue after the first, second review processes. Each manuscript selected was blindly reviewed by at least three reviewers consisting of guest editors and external reviewers.

With increasing demands for indoor GIS, indoor routing and analysis attracts attention from both GIS and architecture worlds. 'Implementing two methods in GIS software for indoor routing: An Empirical Study' aims to provide executable methods in GIS software e.g. ArcGIS for indoor path generation and to explore the possibilities for further analysis. In this paper, two methods are proposed and implemented: Mesh and TIN. The Mesh method used a

Zhihan Lv

lvzhihan@gmail.com

Chen Zhong

c.zhong@ucl.ac.uk

Dingde Jiang

jiangdingde@ise.neu.edu.cn

1 SIAT, Chinese Academy of Science, Shenzhen, China

2 University College London (UCL), London, UK

3 Northeastern University, Shenyang, China 
standard-sized grid graph as the referencing network for a floor and subsequently mapping the movement on a 2D plane to the movement along grid edges. On the other hand, TIN method utilized the TIN as the base, it generates a usable path network by applying two customized TIN. Considering the outputs, the result shows a value for both applying the methods in real use and research analysis of network in indoor environment. TIN provide a network suitable for indoor navigation but need less storage space compared to the Mesh method which provide more accurate network but required extra storage spaces.

Aircraft is a typical transportation facility and its development need to refer to the existing knowledge. With the rapid increase of knowledge, a knowledge map may deliver excess knowledge to users that they cannot manage at once, thereby causing the problem of knowledge overload. Hence, in 'A Novel Method for Adaptive Knowledge Map Construction in the Aircraft Development' a novel method for adaptive knowledge map construction was proposed to solve this problem. To verify the effectiveness of the proposed method, the constructed knowledge map was applied in an airplane wing design to assist users in browsing the knowledge base. Results indicate that the proposed method can change the displayed contents according to user requirement and identify the displayed knowledge nodes at a highly acceptable level, the constructed knowledge map can guide users efficiently, and the knowledge overload can be reduced significantly.

Smart transportation plays an important role in building smart cities. It can obtain mass data from multi-source and use it to manage transportation in an intelligent way. Images and videos can be easily obtained from various sensors in modern road system. They offer abundant information about the transportation. Therefore, visual analysis is a key point in smart transportation management. 'Robust visual tracking using adaptive local appearance model for smart transportation' proposes a robust visual object tracking algorithm using adaptive local appearance model, which can be applied to transportation system. As the main challenge of tracking is to adapt to the target's appearance change, the model is built with a local patch dictionary which is composed of a static part and an online updated part. The updating scheme is important to determine the quality of tracking results.

With the rapid development of urban smart transportation, automatic fare collection system becomes more and more important. The related device based on recognition system in the smart transportation is a key equipment of automatic fare ticket system. It is part of the automatic fare collection system and passenger interface. Because of the complexity of the passenger traffic, how the intelligent recognition system can identify the passengers is a formidable challenge. 'Design of Intelligent Recognition System Based on Gait Recognition Technology in Smart Transportation' designs a sort of intelligent recognition system based on the simplified human gait recognition algorithm. This system can effectively reduce the recognition system's judgment and computation time, and improve the accuracy of judgment. Therefore, the proposed system has a certain application value.

There are a lot of complex environments in real scene, such as illumination variation, shadow variation, object occlusion, which will directly affect the performance of video synopsis. 'A time-slice optimization based weak feature association algorithm for video condensation' adopts Grid Background Model as object detection algorithm, proposing algorithm based on weak feature to solve the object occlusion problem. The experimental result shows that, the method mentioned above can provide a new approach for solving the occlusion problems of video condensation, and have better visualization of abstract video, and achieve up to 6 times concentration to the original video.

It is of great importance in smart transportation surveillance to track object reliably from multimedia streaming data. Sparse representation based target tracking methods often suffer from tracking failure when target is under occlusions, pose changes or illumination changes conditions. 'Tracking in Multimedia Data via Robust Reweighted Local Multi-Task Sparse 
Representation for Transportation Surveillance' proposes a novel robust reweighted local multi-task sparse tracking algorithm. In the algorithm, local patches of all candidate targets are represented as a linear combination of the corresponding local patches from the template dictionary. Observing that the edge of the candidate object contains background information, this paper gives a lower weight coefficient to the reconstruction error regularization located in the edge of the local patches than the middle local patches. Experimental evaluations on challenging sequences demonstrate the effectiveness, accuracy and robustness of our proposed algorithm in comparison with state-of-the-art algorithms.

This paper builds a weighted bus adjacency-stop complex network model of Qingdao city based on the dynamic travel time between each two adjacent stops, using bus system data from 2014.09 to 2015.09, including 261 lines, 1758 stations and 1 billion arrival time of all the buses, provided by Urban Public Transport Development Research Institute of Qingdao. Based on this model, 'Modeling and analysis of bus weighted complex network in Qingdao city based on dynamic travel time' have analyzed the static topological properties of Qingdao bus network, and also studied the features of dynamic weighted network based on average travel time. Weighted average path length shows the characteristics of morning and evening peak in working days, but the standard deviation is $4 \mathrm{~min}$, which reveals the difference of bus system running status between the peak and non-peak is not obvious. Combined with GIS, in this paper, temporal and spatial visual analyses for node strength, edge weight, and weighted average path length in a working day are carried out. The results prove that the morning and evening peak of Qingdao bus system mainly appears in the central areas of Shinan District and Shibei District, and the nodes with large carrying capacity are mainly distributed in the commercial activity center, train station and bus station, etc.

Congestion prediction can support traffic departments to take effective traffic management measures, and it aids users to adopt smarter trip strategies, including route and departure time selection. 'Real-time congestion prediction for urban arterials using adaptive data-driven methods' proposes an adaptive data-driven real-time congestion prediction method. The method framework includes a traffic pattern recognition algorithm based on the adaptive Kmeans clustering, a two-dimensional speed prediction model and an adaptive threshold calibration method. After the principal component analysis, the adaptive K-means cluster algorithm is conducted to obtain different traffic patterns. Congestion recognition is realized with an adaptive threshold calibration method and congestion prediction is then raised according to different traffic patterns. Parameter calibration and model evaluation are carried out on the proposed method using floating car travel speed data of Dongfeng East Road, Guangzhou, China. The results show that the adaptive K-means cluster recognition algorithm can better recognize the traffic patterns than Gaussian Mixture model, and the proposed congestion prediction method has better real-time performance, accuracy and robustness than Autoregressive Integrated Moving Average model and Kalman Filtering method.

Smart transportation has a significantly impact on city management and city planning, which has received extensive attentions from academic and industrial communities. Different from omni-directional sensing system, as a directional sensing system, the multimediadirectional sensor network holds the special coverage scheme, which is usually used for smart cities, smart transportation, and harsh environment surveillance, for instance, nuclear-pollution regions where are inhospitable for people. 'Coverage-Enhancing Approach in Multimedia Directional Sensor Networks for Smart Transportation' advances Virtual Stream Artificial Fish-swarm based Coverage-Enhancing Algorithm (VSAFCEA) as a coverage-enhancing means in multimedia directional sensor networks. Based on comparison of these simulation results (results of presented VSAFCEA and that of other typical coverage-enhancing ways in 
directional sensor networks), the conclusion can be drawn that VSAFCEA could attain higher area coverage rate of directional sensor networks with fewer iterative computing times.

In 'A Novel Rotational Symmetry Dynamic Texture (RSDT) based Sub Space Construction and SCD (Similar-Congruent-Dissimilar) based Scoring Model for Background Subtraction in Real Time Videos', a novel 'SCD (Similar-Congruent-Dissimilar) based scoring” model is used to estimate appearance consistency and temporal coherence of the video. The two-layer approach is used to solve sudden illumination changes and to improve processing speed by adopting mean values of blocks. It is suitable for both indoor and outdoor environments because the subspace construction is purely based on directional codes with rotational symmetry and line symmetry. Metric F-Score is used to measure the performance of the proposed method. Finally this paper provides an effective and robust background subtraction scheme. From performance evaluation, it is observed that Rotational Symmetry Dynamic Texture with SCD based scoring model guarantees accurate background subtraction than the existing literature.

In 'Big Data-Based Multimedia Transcoding Method and Its Application in Multimedia Data Mining-Based Smart Transportation and Telemedicine', the method and system proposed in this paper obtain different data and same data between current multimedia data and pre-stored data by comparing current multimedia data and pre-stored data and encode the attribute information of same data from encoding big data. It is not necessary to encode all multimedia data, but to encode different data and attribute information only. Different data account for a small proportion of the entire multimedia data, while same data represent most of the entire multimedia data. Besides, the encoding of same data is concerned with the attribute information of same data, so the quantity of encoding data is very small and hence the compression ratio is very higher.

Current researches demonstrate that the correlation between property crime and factors, like urban transport, network density, floating population and economic indicator, is significant. 'Spatial-temporal Forecast Research of Property Crime under the Driven of Urban Traffic Factors' confirms that the GA-BP neural network model based on GIS is a reasonable forecast model in the field of property crime forecast research. Given its features, the GA-BP neural network model can forecast other types of rational crime and can set the time window and spatial dimension to obtain the corresponding forecast result based on the accuracy of input data.

Security and privacy are both fundamental requirements of vehicular networking applications. Existing anonymous authentication protocols entail serious performance issues due to serialized verification operations. 'An Efficient Anonymous Authentication Protocol Using Batch Operations for VANETs' presents an efficient anonymous authentication protocol based on signature with message recovery. Different from existing solutions, the proposed protocol authenticates multiple signatures using batch operations. Therefore, the solution can remarkably reduce the total authentication time. Meanwhile the transmission overhead can also be considerably decreased due to the combination of signature and message. Through extensive analysis and simulations, it shows that the proposed protocol outperforms existing ones in terms of communication and computation efficiency.

'QuickSpot: a Video Analytics Solution for On-Street Vacant Parking Spot Detection' introduces QuickSpot, a car-driven video analytics solution for on-street vacant parking spot detection designed as a motion detection, object tracking and visual recognition pipeline. One of the main features of QuickSpot is its simplified setup, as it can be trained on external databases to learn the appearances of the objects it is capable of recognizing (pedestrians and vehicles). To test its performance under different daytime lighting conditions, the trade-off between the accuracy and the computational complexity of QuickSpot with an eye to its practical applicability has been evaluated. The results show that QuickSpot detects parking spot status with an average accuracy 
close to $99 \%$ at a 1-s rate regardless of the illumination conditions, outperforming in an indirect comparison the other car-driven approaches reported in the literature.

With the rapid development of urbane-centered economy, today swelling cities is being filled with massive socioeconomic activities, and urban area have gone through strong but heterogeneous sprawl. Marketer has to put more resources for the ground-based promotion of their products in such metropolis. Therefore, it is very significance to help marketer choose a number of more-suited districts in urban area for promotion. 'The location selection of groundbased promotion in city using human mobility data' investigates location selection problem of ground-based promotion in city with urban computing's perspective. In a provincial capital of China, massive call logs from millions of people have been used to abstract human mobility patterns and urban area have been segmented to hundreds of districts so that we model this problem with two essential cases and give corresponding solutions via greedy algorithms. This application framework can be used in ground based promotion of many application scenarios such as 020 applications that fusing of multisource mobility data.

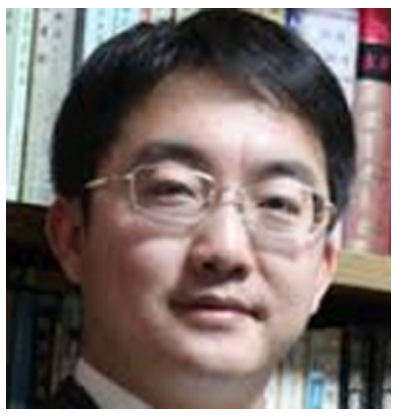

Zhihan Lv is an engineer and researcher of virtual/augmented reality and multimedia major in mathematics and computer science, having plenty of work experience on virtual reality and augmented reality projects, engage in application of computer visualization and computer vision. His research application fields widely range from everyday life to traditional research fields (i.e. geography, biology, medicine). During the past years, he has finished several projects successfully on PC, Website, Smartphone and Smartglasses. In 2012, he was granted $\mathrm{PhD}$. degree in Computer applied technology from Ocean university of China (2006-2012). Before that, he has enjoyed sixteen months full-time research experience at Centre national de la recherche scientifique(CNRS)UPR9080 in Paris (2010-2011). After then, he has fulfilled two-year postdoc research experience at Umea university and a short invited teaching experience at KTH Royal Institute of Technology in Sweden. Since 2012, he has held an assistant professor position at Chinese Academy of Science. 


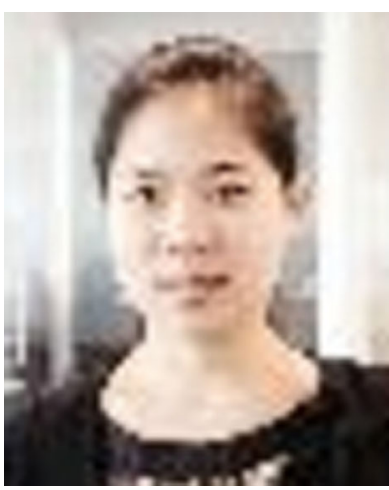

Chen Zhong is a research associate at Centre for Advanced Spatial Analysis (CASA) at University College London (UCL). She is awarded doctoral degree from ETH Zurich, Department of Architecture, ETH Zürich. Her research is on spatiotemporal analysis, modeling and visualization, transportation data analysis, parametric modeling, urban simulation.

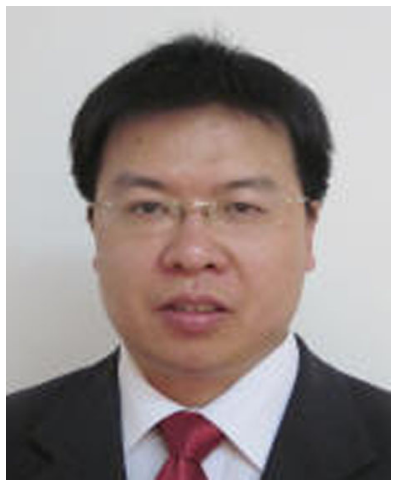

Dingde Jiang is a Professor in the College of Information Science and Engineering at Northeastern Southern University, Shenyang, China. He received the Ph.D. in Communication and Information Systems from University of Electronic Science and Technology of China, Chengdu, China, in 2009. From 2013 to 2014, he was a Visiting Professor with the Department of Computer Science and Engineering at University of Minnesota, Minneapolis, MN, USA. His research focuses on network measurement, modeling and optimization, performance analysis, network management, network security in communication networks, particularly in software defined networks, information-centric networking, energy-efficient networks, and cognitive networks. His research is supported by the National Science Foundation of China, the National Key Technology Research and Development Program of the Ministry of Science and Technology of China, the National High Technology Research and Development Program of China, the Program for New Century Excellent Talents in University of Ministry of Education of China, the Specialized Research Fund for the Doctoral Program of Higher Education of China, the China Postdoctoral Science Foundation, the Scientific Research Foundation of the Education Department of Liaoning Province, China, the Fundamental Research Funds for the Central Universities of China. Dr. Jiang has published over 110 scientific/technical articles. He has authored 1 books and 1 book chapter. He has applied for 12 China Invention Patents. He has been awarded 8 authorized China Invention Patents and 6 software copyrights. He has been serving as an Editor for 1 international journals. He served as a technical program committee (TPC) member for several international conferences. He has received the Best Paper Awards at the International Conferences. 